\title{
Tailored interventions to assist smokers to stop smoking using e- cigarettes (TASSE): Study protocol
}

Catherine Kimber ${ }^{1}$, Daniel Frings ${ }^{1}$, Sharon Cox ${ }^{2}$, Vassilis Sideropoulos ${ }^{2}$, Felix Naughton ${ }^{3}$, Jamie Brown ${ }^{2}$, Hayden McRobbie $^{4}$, Lynne $^{1}$ Dawkins $^{1}$

1 London South Bank University

2 University College London, University of London

3 University of East Anglia

4 University of New South Wales

Funding: The author(s) received no specific funding for this work.

Potential competing interests: The author(s) declared that no potential competing interests exist.

\section{Abstract}

Background: There is increasing evidence that e-cigarettes (EC) are an effective smoking cessation aid when combined with behavioural support. There is further evidence for digital tailored interventions as cost effective approaches that can increase smoking cessation rates. Experimental work also suggests the addition of a 'nicotine fact sheet' can improve smokers' risk perceptions related to EC. However, multifaceted approaches to deliver 'tailored advice' for smoking cessation combining various key evidenced based components are lacking. The aim of this study is to use a Multiphase Optimisation Strategy (MOST) to determine which of five, or a combination thereof, ECorientated intervention components is associated with self-reported cessation over the previous 4-weeks at 12-week follow-up.

Methods/Design: This online study will utilise a 2x2x2x2x2 factorial design resulting in 32 experimental conditions. The five intervention components will be: 1: tailored advice on EC device; 2: tailored advice on e-liquid nicotine strength; 3: tailored advice on e-liquid flavour; 4: e-cigarette written information; 5: text message support. A sample of $\mathrm{N}=1184$ adult, UK resident smokers will be randomly allocated to one of the 32 conditions, which will be permutations of the 5 components (counter-balanced). The primary outcome is 4 weeks of self-reported complete abstinence at 12 weeks post randomisation. Secondary outcomes are 7-day point prevalence, $50 \%$ reduction in baseline cigarettes smoked per day, time to switch and adherence to recommendation. The primary analysis will be by intent-to-treat with the assumption that missing equals smoking. Logistic regression will be used to model the five main effects and the ten $2 \times 2$ interactions. A number of secondary analyses will also be conducted including models adjusting for demographic and smoking indices and including only those who received the intervention.

Discussion: To date, components that assist quitting by use of EC or other aids have largely been studied in isolation. This study presents the first attempt to combine evidenced based interventions, using the MOST method, to test which components are associated with quitting. The findings will be used to inform which components to include and their estimated effect sizes for a definitive randomised controlled trial (RCT) to examine the efficacy of the intervention compared with usual care (own choice and no support).

Keywords: Digital interventions; Tailored Advice; Smoking cessation; Smoking reduction; E-cigarettes; Multi-phase Optimisation Strategy (MOST); Tobacco; Nicotine; Vaping.

\section{Background}

Smoking remains one of the most preventable causes of disease and early death; although its prevalence is in decline, it causes over 7.1 
million deaths worldwide annually (1) including 77,900 in the UK (2). Most smokers report wanting to quit and around a third make a quit attempt each year (3). Of those wanting to quit, approximately half will try to do so unassisted and consequently, long term cessation rates remain extremely low (4). In some countries, there remains policy interest in evidence-based innovations to support quitting including the use of less harmful nicotine products such as e-cigarettes (EC).

The use of EC (also known as 'vaping') is now the most popular method for smoking cessation in England (4) with evidence of efficacy for smoking cessation $(5,6)$ and health improvements where their use has completely replaced smoking $(7,8)$. However, in England since 2016, EC prevalence has plateaued, and, whilst large numbers of smokers (approximately 3 in 5) report having tried EC, most (65\%) fail to maintain use in favour of continuing smoking (9). Aside from dissatisfaction (9) and increasing harm misperceptions (4,9-11), the perceived complexity and technicalities associated with EC use have all been reported to be a hurdle for many smokers $(9,12,13)$ which may impede continued use.

A randomised controlled trial (RCT) showed that an EC in combination with behavioural support provided by a stop smoking service (SSS), is twice as effective compared with nicotine replacement therapy alongside behavioural support (6). However, the percentage of smokers accessing SSS in England remains low (14) and access to vaping products through SSS is currently limited. Survey data suggest that most purchases are made via specialist vape shops (39\%) or online (16\%) (15). Successful switchers (from smoking to exclusive EC use) often report that finding the right device, nicotine strength and flavour are essential (16). However, whilst many vape shops offer technical and behaviour support surrounding switching to EC, this is often absent when making online purchases. Furthermore, where support and advice is available, this can feel overwhelming and leave some smokers describing the vape shop environment as intimidating (17). Thus, there may be utility in assisting smokers who make e-cigarette purchases online, by providing simple tailored advice on which items to choose and, offering support around e-cigarette use.

Brief tailored interventions (18) including mobile and web-based support have been found cost effective, wide reaching and targeted approaches that can increase smoking cessation rates $(19,20)$. More recent experimental work suggest that written information such as a nicotine fact sheet may be a promising promoting tool to correct risk perceptions related to EC (21). Messages conveying reduced harm information have also been associated with lower odds of smoking (22). This is important since an increasing number of the population in England consider EC to be equally (over $45 \%$ ) or more harmful (over $20 \%$ ) than smoking (23) and, safety concerns are frequently cited among smokers (23\%) as a reason for not trying EC (9). Multifaceted approaches which deliver tailored advice combining various key components for effective interventions are lacking. Given the lack of advice and existing misperceptions around EC, this could be one avenue to increase switching to vaping and promote long-term smoking abstinence.

The multiphase optimisation strategy (MOST) provides a useful framework to develop improved treatment or programme packages based on existing programme components $(24,25)$. MOST consists of three phases: preparation, optimisation and evaluation, within which, similarly to traditional RCT, in the preparation phase, appropriate components are identified and selected to form part of an intervention. In addition, an optimisation phase is introduced to assess the performance of each intervention component as well as the outcomes of their interactions with other components (i.e. main effects and interactive effects). In the optimisation phase, weak or non-performing components are excluded from the final refined intervention package that will eventually be examined via a RCT. In this study (the optimisation stage), a multi-factorial design will be used to test the effectiveness of a novel, scalable online intervention to promote EC use to achieve complete smoking cessation. The components will be brief, inexpensive, and involve no face-to-face contact. Tailored advice on choice of: (1) EC device; (2) eliquid nicotine strength; and (3) e-liquid flavour will be constructed to match with nicotine/cigarette dependence, current smoking behaviours and preferences. We will also incorporate a fourth component (4) written information around the reduced harm of vaping compared to smoking using Cancer Research UK infographic materials and (5) technical and behavioural support around stopping smoking and using EC to support quitting, sent by mobile phone text messages (these will be developed and tested in a pilot study as part of this project). The control arm will include a $£ 50$ voucher to purchase an EC online only but no further intervention components (i.e. the arm where all interventions are 'off'). Experimental arms will include a free EC kit (up to the value of £50) and a combination of the various interventions 
under investigation. In doing so, this study will assess which component or combination of components are associated with greater proportion of complete abstinence and smoking reduction and, what effect size could be expected, in order to inform a definitive RCT with longer-term outcomes to examine the efficacy of the intervention compared with usual care (own choice and no support). The findings will provide proof of principle for the intervention package and, also identify the component(s) that best promote cessation.

\section{Aims and Objectives}

The overarching aim of this study is to use the Multiphase Optimisation Strategy (MOST) to determine which of five, or a combination thereof, intervention components (1: tailored advice on device; 2: tailored advice on nicotine strength; 3: tailored advice on flavour; 4: e-cigarette written information; 5: text message support) is associated with the best chances of making a complete switch to vaping.

\section{Primary Objective:}

To determine which intervention component or components is associated with the greatest proportion of smokers who self-report successful abstinence from smoking (not a single puff) over the previous 4-weeks at 12-weeks post-randomisation.

\section{Secondary Objectives:}

To examine the differences between intervention components in regard to:

1. 7-day point-prevalence abstinence at 12 weeks post-randomisation,

2. Proportion of people reporting a $50 \%$ reduction or more in baseline cigarette consumption at 12 weeks post-randomisation in those who did not achieve full abstinence,

3. Time (in days) to switch completely from smoking to vaping (i.e. stop smoking), and

4. To present data on adherence and compliance to the tailored recommendations around EC device, nicotine strength and flavour.

\section{Intervention components}

\section{Tailored advice}

1. Advice on device will be tailored using three five-point Likert-type scale items with the options "strongly disagree to strongly agree" scoring from 1 to 5 : i) "The e-cigarette must be small", ii) "I prefer to be able to see lots of vapour, including when exhaling" (reverse-scoring 5 to 1 ) and iii) "The technicalities of the e-cigarette put me off". Participants scoring between 3 and 7 will be assigned a tank system e-cigarette device (Arc 5) which is typically associated with greater volume of aerosol, those scoring between 8 and 11 will be assigned the tank system pen-like middle range device (Tornado EX2). Scores above 12 will be assigned a refillable pod-system e-cigarette (Skope-P) which is characterised by its small, discrete and easy to use features. This choice of devices was informed by several factors. For greater control and consistency, it is important that all devices are as aligned as possible in terms of i) price to minimise risks of non-compliance with regards to the tailored device selection advice, ii) compatibility with the range of e-liquid formulation and e-liquid nicotine strengths on offer by the vendor. To minimise risks of introducing barriers such as technicalities, all devices will be starter kits and no sub-ohm devices (device with an atomiser resistance of 0.05 ohms or lower) will be included due to their incompatibility with high nicotine strengths and complex technical features.

2. Advice on nicotine strength will be determined from participants' answers to the question 'How soon after waking do you smoke your first cigarette?' an item making up both the Fagerström Test for Cigarette Dependence [FTCD] (26) and Heaviness of Smoking Index [HSI] (27). Time to first cigarette after waking has been identified as the best predictor of tobacco dependence and therefore a robust means of tailoring advice on nicotine strength (28). Response options include $\leq 5 \mathrm{mins}$ scoring 3,6 -30mins gives a score of 2 , 31-60mins a score of 1 , 
and after 60 mins gives a score 0 . Participant scoring 3 or 2 will be assigned to $18 \mathrm{mg} / \mathrm{mL}$, those scoring 1 and 0 will be assigned a $14 \mathrm{mg} / \mathrm{mL}$ and $10 \mathrm{mg} / \mathrm{mL}$ nicotine strength, respectively.

3. Advice on flavours will be tailored using the following: "Do you smoke more menthol cigarettes than regular tobacco cigarettes?". Those answering 'Yes' will be assigned menthol flavour e-liquid, and those answering 'No' will be directed to a question using items that assess taste preferences (i.e. "In your attempt to quit smoking, do you want something that tastes like smoking or a complete change?" - with the options "Yes I want something that tastes like smoking" which will result in being assigned tobacco and "No I want a complete change" will result in being assigned fruit flavour).

Tailored advice on device, nicotine strength and flavour will be displayed right before the end of the baseline survey and sent via email.

\section{Written information about E-cigarettes}

The fourth component 'written information' is intended to provide factual information about vaping in order to allay safety concerns and misperceptions of harm. We will use the Cancer Research UK one-page infographic around the reduced harm of vaping compared to smoking. Permission has been granted by CRUK for its use for the purpose of this study. Examples include "Tobacco is the biggest cause of preventable death in the UK", "Growing evidence shows e-cigarettes are helping people to stop smoking". Recent evidence suggests that CRUK EC infographics are credible and increase perceptions that EC are an effective cessation aid (29). Thus, this parsimonious infographic could be a persuasive communicating tool. Participants allocated $\mathrm{ON}$ for this component, will receive this via email upon completing the baseline survey.

\section{Technical and behavioural support via text messages}

Although there exists a bank of behavioural text messages for smoking cessation support (30), there is no comprehensive collection related to cessation while using an EC. Through extensive public and professional engagement, a set of 99 text messages has been developed for this study which cover both technical and behavioural aspects of e-cigarette use (to keep their device with them, have a spare battery handy, refill their device before going out, e.g. "If you have a cough, don't worry - it's your lungs starting to get rid of the tar that has built up over the years from smoking. It's all progress!", "aim to stop smoking completely", "Some pubs and bars are vape friendly. Check with staff who might be happy to let you vape indoors"). This includes some general messages for smoking cessation support taken from the iQuit in Practice message bank (31). An initial set of texts have been developed and tested through a pilot study with $\mathrm{N}=127$ smokers, vapers and exsmokers who rated how the text messages on their understandability, believability, usefulness and clarity. Following work with our PPI group, we then selected a final 70 messages for inclusion and determined the order in which they will be sent (the full procedure and list of final messages will be made publicly available and written up separately). The 70 messages plus two instructional text messages will be sent using Simple Mail Transfer Protocol (SMTP) via PageOne Communications LTD: twice daily for two weeks, one a day for four weeks, then every other day for four weeks, finally one a week for 2 weeks. These will be sent to those allocated to the text message condition ON for the technical and behavioural support.

\section{Participants}

\section{Recruitment}

This online study will be advertised through social media, radio adverts and the distribution of flyers and posters. We will recruit smokers who are interested in stopping smoking and will continue to recruit until 1184 vouchers are redeemed to minimise the impact of dropouts (see section on sample size calculation).

Inclusion/exclusion criteria 
Participants will have to meet the following criteria for participation: aged 18 or over, daily smoker, UK resident, fluent in English, interested in quitting, interested in using EC, currently have access to a mobile phone, being able to make an online purchase. Participants will be unable to participate if any of the following apply: under 18 years old, currently using an EC daily (exclusively or dual user), non-UK resident, noninterested in making a quit attempt, not interested in using an EC, have no access to a mobile phone, unable to make an online purchase, unable or unwilling to be contacted in 3 months-time, affiliation with the tobacco or e-cigarette industries.

\section{Study population/ Sample size}

Recent data from Holliday (32), where the intervention arm was given brief advice to quit smoking and provided with an EC and information on how to use it, showed a 6 -month abstinence rate of $15 \%$. This intervention arm received a little more than the control group will receive in our study (EC without any advice or information) and so we estimate a 3-month abstinence rate of $16 \%$. We estimate that tailoring on nicotine strength, flavour, or device could increase abstinence rates by $30 \%$, however the lowest performing component is expected to be written information which we estimate to increase abstinence rates by $20 \%$ (33). To detect two-tailed differences between 16\% (control) and 19\% (EC Info) with a power of 0.80 at $\alpha=0.05$, a total sample of 1184 is required. This sample size allows the detection of differences between two cells of $2 X 2$ interactions (total $n=592$ ) with $O R>=1.340 .75$ with the same power/ $\alpha$ parameters. The sample size was revised and increased to $\mathrm{N}=1334$ (in May 2020) to replace instances of protocol violations. Here, we define protocol violations as multiple completions from the same individual, these include participants who completed the baseline questionnaire in its entirety multiple times thus were randomised to more than one condition. On every instance, only the first entry from that individual will be included in the intention-to-treat analysis and all subsequent entries from that same individual will be removed.

\section{Measures}

Demographic variables will include age, gender, ethnicity, occupation, highest qualification and postcode. Smoking characteristics and history will be assessed by measuring heaviness of smoking using the HIS measured at baseline and at follow-up (number of cigarettes smoked per day, time to first cigarette and years smoked), numbers of previous quit attempts and any smoking cessation aids used in the past.

Motivation to quit smoking and self confidence in succeeding will be measured using the Motivation to Stop Scale [MTSS] and by asking "How confident do you feel in being able to quit for good?" on a 5-point Likert-type scale.

The validated single item questionnaire Self-investment and Self-definition [SISI] (34) will assess social identity in relation to smoker, vaper, dual user and non-smoker identities using "I identify as a [...]" on a 7-point Likert-type scale ("strongly agree to strongly disagree"). Social identity will be assessed at baseline and at follow-up.

History of vaping will be assessed by asking about previous experience of EC use, reasons for not using or for discontinuing use where relevant (registered and reported separately).

Perceptions of harms associated with e-cigarettes will be measured using a single item 6-point Likert-type scale ("Compared to tobacco smoking, how harmful do you think electronic cigarettes are?" with the options "Much more harmful" to "a lot less harmful") adapted from the Action on Smoking and Health (ASH) survey (35).

\section{Outcome variables}

Primary outcome:

1. The proportion of people who report complete abstinence from smoking over the previous 4-weeks at 12-weeks post randomisation. Secondary outcomes: 
1. The proportion of people who report complete abstinence from smoking over the previous 7 days at 12-weeks post randomisation.

2. The proportion of people who report $50 \%$ or greater smoking reduction in baseline cigarette consumption at 12 weeks post-randomisation in participants who did not achieve full abstinence.

3. Time (in days) to switch from smoking to complete vaping (i.e. self-reported no smoking for at least 7 days) in those who report complete abstinence from smoking over the previous 4-weeks at 12-weeks post randomisation

4. Adherence to recommendation (Yes vs. No)

\section{Study settings/Procedure}

From the study webpage, participants will be directed to the Qualtrics baseline survey to check their eligibility. Eligible participants will be invited to provide electronic informed consent before completing the baseline survey. After completing their baseline measures, participants will be randomly allocated to one of the 32 conditions (permutations of ON and OFF components of the intervention, see Table 1). For example, for the following permutation: tailored advice for i) device ON, ii) nicotine strength ON, flavour OFF, written information OFF, text messages ON, participants will be advised to select a specific EC device and nicotine strength (based on their baseline questionnaire responses) and receive text messages on technical and behavioural support, they will not receive advice on flavour and written information. Conditions will be randomised in Qualtrics using the Randomizer function. Randomisation will occur after participants completed the baseline measures and provided their contact details. Thereafter, Qualtrics will randomly present 1 of the 32 conditions - each generated equal number of times. Each recommendation will be displayed at the end of the baseline survey and, sent via email along with a direct link to the EC store and their unique voucher code to make their online purchase. Purchasing data including study voucher activation for each participant will be recorded by the EC store and transferred through a secure passcode protected data repository to be cross-referenced prior to analysis.

After 3 months, participants will be emailed with a link to complete the follow-up questionnaire. Up to 5 reminders will be sent for this purpose. The follow up questionnaire will ask about abstinence and cigarette smoking (cigarettes smoked per day in the last 7 days), EC use, compliance, and helpfulness of the different intervention components. Those who do not complete the follow up survey will be e-mailed with a simple question 'Have you smoked at all in the last 4 weeks' to allow us to capture data for our primary outcome from as many participants as possible.

\section{Design and Statistical analysis}

\section{Primary analysis}

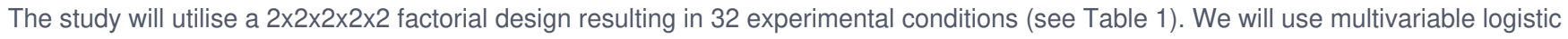
regression to model the primary outcome: complete abstinence over the last 4-weeks at the 12-weeks follow up on an intention to treat basis - that is, all participants who are randomised will be included in the analysis with the exception of those classified as protocol violations (i.e. who completed the baseline survey more than once). Participants lost to follow up will be coded as smoking in line with this aspect of the Russell Standard of smoking (although we will not biochemically validate the self-report at this stage). Abstinence will be defined as selfreported no smoking (not a single puff) for the previous 4-weeks (regardless of current e-cigarette use). All effects will be tested at $p<.05$. The model will include the five main effects (each coded using effect coding: $-1=$ off and $+1=0$ ) and all ten 2 -way interactions. It is not necessary to explore the three way and higher interactions for the purposes of deciding whether to retain an intervention component.

\section{Secondary analysis}

Given that the primary approach will include a number of participants who did not actually receive the intervention, secondary analysis will repeat the above model but include only those who redeemed their voucher (i.e. who proceeded to making their online purchase for a EC kit; 
data which we will capture). We will also exclude people who self-report complete abstinence but never used the EC.

The above analyses will also be repeated, including age, gender, ethnicity, socio-economic status (SES), MTSS and HSI as covariates. Finally, we will run another model including only participants who completed the 12-weeks measure (sample size allowing).

We will retain statistically significant components in a definitive RCT if the Bayes factor $>2$. If a component fails to reach this threshold and its efficacy could not be improved upon, this component would not be included in a definitive RCT.

The same models will be repeated to assess the secondary outcomes 1 (7-day point prevalence at the 12 weeks follow up) and 2 (50\% reduction in smoking). Secondary outcome 3 (time in days to switch from smoking to vaping in those who achieved complete abstinence) will be explored using a one-way ANOVA to explore differences between the five intervention components. Given that this will include only those who achieved complete abstinence, only main effects will be explored.

Adherence to recommended product, flavour and nicotine strength, and engagement with written information and text messages (secondary outcome 4) will be presented using frequencies and descriptive statistics.

Table 1. Permutations for the 5 components of the intervention: tailored advice on 1) EC device, 2) Nicotine strength, 3) flavour, 4) Written information, 5) Text messages support 


\begin{tabular}{|c|c|c|c|c|c|}
\hline Permutations & EC Device & Nicotine strength & Flavour & Written info & Text messages \\
\hline 1 & On & On & On & On & On \\
\hline 2 & On & On & On & On & Off \\
\hline 3 & On & On & On & Off & On \\
\hline 4 & On & On & On & Off & Off \\
\hline 5 & On & On & Off & On & On \\
\hline 6 & On & On & Off & On & Off \\
\hline 7 & On & On & Off & Off & On \\
\hline 8 & On & On & Off & Off & Off \\
\hline 9 & On & Off & On & On & On \\
\hline 10 & On & Off & On & On & Off \\
\hline 11 & On & Off & On & Off & On \\
\hline 12 & On & Off & On & Off & Off \\
\hline 13 & On & Off & Off & On & On \\
\hline 14 & On & Off & Off & On & Off \\
\hline 15 & On & Off & Off & Off & On \\
\hline 16 & On & Off & Off & Off & Off \\
\hline 17 & Off & On & On & On & On \\
\hline 18 & Off & On & On & On & Off \\
\hline 19 & Off & On & On & Off & On \\
\hline 20 & Off & On & On & Off & Off \\
\hline 21 & Off & On & Off & On & On \\
\hline 22 & Off & On & Off & On & Off \\
\hline 23 & Off & On & Off & Off & On \\
\hline 24 & Off & On & Off & Off & Off \\
\hline 25 & Off & Off & On & On & On \\
\hline 26 & Off & Off & On & On & Off \\
\hline 27 & Off & Off & On & Off & On \\
\hline 28 & Off & Off & On & Off & Off \\
\hline 29 & Off & Off & Off & On & On \\
\hline 30 & Off & Off & Off & On & Off \\
\hline 31 & Off & Off & Off & Off & On \\
\hline 32 & Off & Off & Off & Off & Off \\
\hline
\end{tabular}

Note. Participants will be randomised to one of the 32 conditions (permutations of ON and OFF components of the intervention). For example, permutation 7: tailored advice for i) device ON, ii) nicotine strength ON, flavour OFF, written information OFF, text messages ON, participants will be advised to select a specific e-cigarette device and nicotine strength (based on their baseline questionnaire responses) and receive text messages on technical and behavioural support. These participants will not receive advice on flavour and written information.

\section{Discussion}

The aim of this study is to evaluate the performance of five components (1: tailored advice on device; 2: tailored advice on nicotine strength; 3: tailored advice on flavour; 4: e-cigarette written information; 5 : text message support) in isolation and in combination that have potential to increase the proportion of smokers, who purchase a vaping product online, to make a complete switch to vaping. This study will provide an estimated effect size to inform which components to include in a full-powered randomised controlled trial (RCT) to examine the efficacy of the intervention compared with usual care. 
Understanding how best to support EC users to quit smoking for good is an important public health goal. EC are now the most popular method for quitting smoking and there is increasing evidence which suggests their effectiveness when used in combination with behavioural support (6) however, the type and level of support needed remains underexplored. Delivering online brief tailored advice on EC device, nicotine strength and flavour, technical support via text messaging may offer a cost effective and wide reaching strategy to help greater proportion of smokers to overcome the technical barriers typically associated with vaping (17), and promote complete switch away from smoking.

The study has been designed to maximise both internal and external validity. Randomisation will be automated so that each permutation will be generated an equal number of times via the survey platform Qualtrics at the end of the baseline measures to minimise the effect on dropout rate when applying the intention-to-treat analysis. To minimise non-compliance rate, the sequence of events, wording for the tailored advice, all were carefully considered. Likewise, there is no discrepancy in participation incentives as all participants receive a voucher for the same value. Extra care was applied when making the choice for the devices and nicotine strengths that will be used for tailoring advice. Device types selected are all classified as starter vape kits and do not include sub-ohms devices which contain more sophisticated technical features and are not compatible with high nicotine strengths. We have also carefully considered consistency between conditions, for example the integration of the online store experience does not deviate from the natural environment and at the same time is consistent between those in the control condition (with all permutations OFF) and those allocated to one or more conditions ON.

However, some limitations must be noted. Outcome measures will be obtained online with no biochemical validation of abstinence which may be subject to biased response. However, although this presents its limitation, requiring saliva samples to verify and measure cotinine levels would likely increase costs significantly and affect response rate even via post. It is worth pointing that this is an early stage study and forms part of the optimisation phase to decide on which intervention to retain in the full RCT. Non-compliance to tailored advice is likely to occur, although some measures were taken in anticipation, purchases will be monitored and a monthly report from the EC retail company will help monitor adherence (or lack of) to the recommendations. Thirdly, we have limited the choice of devices and nicotine strength to 3 , this choice was informed by the literature and feedback form our PPI and for the reasons previously highlighted. However, many more device types and nicotine strengths range are currently available in the market and will be available to those in the control arm. We were unable to tailor advice on the nicotine formulation ingredients notably the ratio of propylene glycol and glycerol, and nicotine salts, due to the incompatibility and cross-over with some nicotine strengths and device types.

This research ultimately aims to maximise the number of smokers who quit smoking using EC using a novel, scalable approach that could easily be adopted by online e-cigarette stores. The study is designed to achieve a high response rate; it is a light touch intervention with no face-to-face contact and at very low burden to participants (short baseline and follow up survey questionnaires). The findings of this study will be used to refine the components and combinations thereof and inform decisions to proceed to a future full well-powered RCT.

\section{Funding details}

This study is funded by the Medical Research Council (MRC/PHIND; Grant Ref: MR/T002352/1).

Potential Conflict of Interests

CK, FN and VS have no conflict of interests. DF was the principal investigator in a randomised controlled trial comparing Allen Carr's Easyway to quit smoking to NHS provision (see https://osf.io/9kj8d/ for funding details, protocols, registrations and outputs). SC provides expert consultancy to providers of UK life insurance on matters relating to smoking cessation. JB has received unrestricted funding from Pfizer to study smoking cessation. HM has received honoria for speaking at smoking cessation educational meetings organised by Pfizer. LD has provided consultancy for the pharmaceutical industry and acted as an expert witness for an EC patent infringement case. 
Authors' contributions

LD is the lead principal investigator and grant holder for this project. Along with DF, SC, VS, FN and JB, LD and HM conceived the original idea for the project, designed the study, refined the methodology and contributed to the grant application. VS contributed to the design, technical expertise including software programming and drafting of the protocol. CK led on the drafting of the manuscript and is responsible for the day-to-day running of the project and data collection. All authors contributed significantly to and edited drafts of this manuscript. All authors have read and approved the final manuscript.

\section{Acknowledgments}

We are thankful to our PPI, Nadine Kapitan, Sarah Jakes, Jessica Harding and James Binnie who contributed to the development and ordering of the text messages.

Authors' details

CK is a Research Fellow at the Centre of Addictive Behaviours Research at London South Bank University. DF is a Professor of Social Psychology at London South Bank University. SC is a Senior Research Fellow at London South Bank University. JB is a Professor of Behavioural Science \& Health and Co-Director of the UCL Tobacco and Alcohol Research Group. FN is a Health Psychologist and a Senior Lecturer in Health Psychology within the School of Health Sciences, University of East Anglia. HM is Professor at the National Drug and Alcohol Research Centre, University of New South Wales and Consultant in Lifestyle Medicine at Lakes District Health Board, Rotorua, New Zealand. VS is a Psychology \& Human Development Research Tech at UCL Institute of Education. LD is a Professor of Nicotine and Tobacco Studies at the Centre for Addictive Behaviours Research at London South Bank University.

Ethical oversight

The study has received full ethical approval from London South Bank University's ethics committee (approval date: 14/01/2020; reference ETH1920-0043). Participants will provide electronic consent prior to completing the baseline survey.

\section{References}

1. The Tobacco Atlas [Internet]. [cited 2020 Feb 18]. Available from: https://tobaccoatlas.org/topic/deaths/

2. NHS NHS. Part 1: Smoking-related ill health and mortality - NHS Digital [Internet]. 2018 [cited 2020 Feb 4]. Available from: https://digital.nhs.uk/data-and-information/publications/statistical/statistics-on-smoking/statistics-on-smoking-england-2018/part-1smoking-related-ill-health-and-mortality

3. West R, Brown J. Latest Statistics - Smoking In England [Internet]. 2018 [cited 2018 Feb 8]. Available from: http://www.smokinginengland.info/latest-statistics/

4. West R, Brown J, Beard E. Trends in e-cigarette use in England [Internet]. 2020. Available from: http://www.smokinginengland.info/downloadfile/?type=latest-stats\&src=11

5. Hartmann-Boyce J, McRobbie H, Bullen C, Begh R, Stead LF, Hajek P. Electronic cigarettes for smoking cessation. In: Hartmann-Boyce J, editor. Cochrane Database of Systematic Reviews [Internet]. Chichester, UK: John Wiley \& Sons, Ltd; 2016 [cited 2017 Jul 11]. Available from: http://doi.wiley.com/10.1002/14651858.CD010216.pub3

6. Hajek P, Phillips-Waller A, Przulj D, Pesola F, Myers Smith K, Bisal N, et al. A Randomized Trial of E-Cigarettes versus NicotineReplacement Therapy. N Engl J Med [Internet]. 2019;NEJMoa1808779. Available from: 
http://www.nejm.org/doi/10.1056/NEJMoa1808779

7. Shahab L, Goniewicz ML, Blount BC, Brown J, McNeill A, Alwis KU, et al. Nicotine, carcinogen, and toxin exposure in long-term ecigarette and nicotine replacement therapy users: A cross-sectional study. Ann Intern Med [Internet]. 2017 Feb 7;24(18):442-8. Available from: http://dx.doi.org/10.7326/M16-1107

8. Campagna D, Amaradio MD, Sands MF, Polosa R. Respiratory infections and pneumonia : potential benefits of switching from smoking to vaping. Pneumonia [Internet]. 2016;8(1):8-11. Available from: http://pneumonia.biomedcentral.com/articles/10.1186/s41479-016-0001-2

9. ASH. Use of e-cigarettes among adults in Great Britain 2017. 2017;(May 2017). Available fromhttp://ash.org.uk/information-andresources/fact-sheets/use-of-e-cigarettes-among-adults-in-great-britain-2017/

10. Wilson S, Partos T, Mcneill A, Brose LS. Harm perceptions of e-cigarettes and other nicotine products in a UK sample. $2019 ; 1-10$. Available from: https://www.onlinelibrary.wiley.com/doi/pdf/10.1111/add.14502

11. Farsalinos KE, Siakas G, Poulas K, Voudris V, Merakou K, Barbouni A. Electronic cigarette use in Greece : an analysis of a representative population sample in Attica prefecture. 2018;1-8.

12. Mckeganey N, Dickson T. Why Don't More Smokers Switch to Using E-Cigarettes: The Views of Confirmed Smokers. Int J Environ Res Public Health [Internet]. 2017;14(6):647. Available from: http://www.mdpi.com/1660-4601/14/6/647

13. Wadsworth E, Neale J, Mcneill A, Hitchman SC. How and Why Do Smokers Start Using E-Cigarettes? Qualitative Study of Vapers in London, UK. 2016;

14. West R, Brown J. Latest Statistics - Smoking In England - STS120720 [Internet]. 2020 [cited 2020 Feb 18]. Available from: http://www.smokinginengland.info/latest-statistics/

15. IFF Research. Understanding the Online E-cigarette market [Internet]. 2016. Available from: www.gov.uk/HMRC

16. Notley C, Ward E, Dawkins L, Holland R. The unique contribution of e-cigarettes for tobacco harm reduction in supporting smoking relapse prevention. Harm Reduct J [Internet]. 2018 Dec 20;15(1):31. Available from:

https://harmreductionjournal.biomedcentral.com/articles/10.1186/s12954-018-0237-7

17. Ward E, Cox S, Dawkins L, Jakes S, Holland R, Notley C. A Qualitative Exploration of the Role of Vape Shop Environments in Supporting Smoking Abstinence. Int J Environ Res Public Health [Internet]. 2018 Feb;15(2):297. Available from: http://www.mdpi.com/1660$\underline{4601 / 15 / 2 / 297}$

18. Gilbert HM, Leurent B, Sutton S, Alexis-Garsee C, Morris RW, Nazareth I. ESCAPE: A randomised controlled trial of computertailored smoking cessation advice in primary care. Addiction. 2013 Apr;108(4):811-9.

19. Scott-Sheldon LAJ, Lantini R, Jennings EG, Thind H, Rosen RK, Salmoirago-Blotcher E, et al. Text Messaging-Based Interventions for Smoking Cessation: A Systematic Review and Meta-Analysis. JMIR mHealth uHealth. 2016 May 20;4(2):e49.

20. Hébert ET, Stevens EM, Frank SG, Kendzor DE, Wetter DW, Zvolensky MJ, et al. An ecological momentary intervention for smoking cessation: The associations of just-in-time, tailored messages with lapse risk factors. Addict Behav. 2018 Mar 1;78:30-5.

21. Yang B, Owusu D, Popova L. Effects of a Nicotine Fact Sheet on Perceived Risk of Nicotine and E-Cigarettes and Intentions to Seek Information About and Use E-Cigarettes. Int J Environ Res Public Health. 2019 Jan 1;17(1).

22. Jo CL, Golden SD, Noar SM, Rini C, Ribisl KM. Effects of E-cigarette Advertising Messages and Cues on Cessation Outcomes. Tob Regul Sci [Internet]. 2018;4(1):562-72. Available from: http://www.ingentaconnect.com/content/10.18001/TRS.4.1.3

23. West R, Beard E, Brown J. Electronic cigarettes in England - latest trends STS140123 [Internet]. 2020. Available from: http://www.smokinginengland.info/downloadfile/?type=latest-stats\&src=11

24. Collins LM, Kugler KC, Gwadz MV. Optimization of Multicomponent Behavioral and Biobehavioral Interventions for the Prevention and Treatment of HIV/AIDS. AIDS Behav [Internet]. 2016 Jan 4 [cited 2020 Feb 18];20(S1):197-214. Available from: http://link.springer.com/10.1007/s10461-015-1145-4

25. Piper ME, Cook JW, Schlam TR, Jorenby DE, Smith SS, Collins LM, et al. A Randomized Controlled Trial of an Optimized Smoking Treatment Delivered in Primary Care. Ann Behav Med [Internet]. 2018 Sep 13 [cited 2020 Feb 18];52(10):854-64. Available from: https://academic.oup.com/abm/article/52/10/854/4858421 
26. Fagerström K. Determinants of tobacco use and renaming the FTND to the Fagerström Test for Cigarette Dependence. Nicotine Tob Res [Internet]. 2012;14(1):75-8. Available from: http://search.ebscohost.com/login.aspx?direct=true\&db=psyh\&AN=2011-30003$\underline{008 \& \text { site }=\text { ehost-live }}$

27. Heatherton TF, Kozlowski LT, Frecker RC, Rickert W, Robinson J. Heaviness of Smoking Index (HSI). Br J Addict [Internet]. 1989;84(7):791-9. Available from: http://lib.adai.washington.edu/dbtw-wpd/exec/dbtwpub.dll?AC=QBE_QUERY\&QY=find \%28AccessNo ct 555\%29 and \%28Status ct public\%29\&XC=/dbtw-

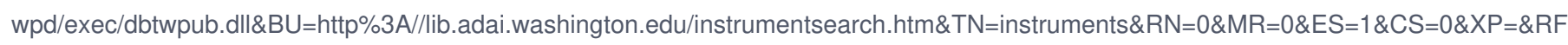

28. Fagerström K. Time to first cigarette; The best single indicator of tobacco dependence? Monaldi Arch Chest Dis - Pulm Ser. 2003;59(1):91-4.

29. Tattan-Birch H, Jackson SE, Ide C, Bauld L, Shahab L. Evaluation of the Impact of a Regional Educational Advertising Campaign on Harm Perceptions of E-Cigarettes, Prevalence of E-Cigarette Use, and Quit Attempts Among Smokers. Nicotine Tob Res. $2019 ; 1-7$.

30. Whittaker R, McRobbie H, Bullen C, Rodgers A, Gu Y, Dobson R. Mobile phone text messaging and app-based interventions for smoking cessation. Vol. 2019, Cochrane Database of Systematic Reviews. John Wiley and Sons Ltd; 2019.

31. Naughton F, Jamison J, Boase S, Sloan M, Gilbert H, Prevost AT, et al. Randomized controlled trial to assess the short-term effectiveness of tailored web- and text-based facilitation of smoking cessation in primary care (iQuit in Practice). Addiction. 2014;109(7):1184-93.

32. Holliday R, Preshaw PM, Ryan V, Sniehotta FF, McDonald S, Bauld L, et al. A feasibility study with embedded pilot randomised controlled trial and process evaluation of electronic cigarettes for smoking cessation in patients with periodontitis. Pilot Feasibility Stud [Internet]. 2019 [cited 2020 Mar 13];5(1). Available from: https://doi.org/10.1186/s40814-019-0451-4

33. Hartmann-Boyce J, Lancaster T, Stead LF. Print-based self-help interventions for smoking cessation. Vol. 2017, Cochrane Database of Systematic Reviews. John Wiley and Sons Ltd; 2014.

34. Postmes T, Haslam SA, Jans L. A single-item measure of social identification: Reliability, validity, and utility. Br J Soc Psychol. 2013;52(4):597-617.

35. ASH. Use of e-cigarettes ( vapourisers ) among adults in Great Britain BACKGROUND - HEALTH EFFECTS OF VAPING COMPARED TO SMOKING. 2018. 